\title{
BANACH LATTICES WITH A WEAK ORDER UNIT
}

\author{
BY CONSTANTIN NICULESCU
}

Communicated by R. G. Bartle, April 22, 1976

Starting from a result due to $H$. Rosenthal (see Lemma 1.3 in [6]), we present a new result which outlines a geometrical condition for the existence of a weak order unit (i.e., a total element) in the dual of a Banach lattice: the nonexistence of a lattice isomorph of a space $l_{1}(\Gamma)$ for $\Gamma$ an uncountable set (see Theorem 2 below). As a consequence we obtain that the dual of a Banach space $E$ having local unconditional structure (as defined in [1] has the Radon-Nikodým property iff $E$ does not contain a copy of $l_{1}$. R. C. James constructed, in [3], an example of separable Banach space $J$ with a nonseparable dual such that $c_{0}$ and $l_{1}$ do not embed in $J$. Consequently $J$ does not have local unconditional structure.

Let $L$ be the class of all Banach spaces $X$ satisfying the following two conditions:

(L1) $X^{\prime}$ is an order complete Banach lattice;

(L2) there is a $v \in X^{\prime \prime}$ such that $x \in X,|x| \wedge|v|=0$ implies $x=0$.

Each Banach lattice with a weak order unit, or each predual of an $L_{1}(\mu)$ space, belongs to $L$.

1. LEMmA. If $X \in L$, then there exists an order complete Banach lattice $E$ with a weak order unit and a lattice isometry $i: X^{\prime} \rightarrow E^{\prime}$ such that: (a) $i\left(X^{\prime}\right)$ is complemented in $E^{\prime}$, and (b) $i\left(X^{\prime}\right)$ is formed by order continuous functionals on $E$.

Hint. Consider for $E$ the band generated by $v$ in $X^{\prime \prime}$.

If $Z$ is an order complete Banach lattice and $A \subset Z$ is a closed subspace, then we shall denoted by $\Sigma(A)$ an order complete closed sublattice of the band generated by $A$ such that $A \subset \Sigma(A)$.

2. Theorem. Let $E \in L$ and let $A \subset E^{\prime}$ be a closed subspace. Then either:

(i) $\Sigma(A)$ has a weak order unit $u^{\prime}>0$; or,

(ii) $A$ contains an isomorph of $l_{1}(\Gamma)$ (for $\Gamma$ an uncountable set) that is complemented in $\Sigma(A)$, and $\Sigma(A)$ contains a lattice isomorph of $l_{1}(\Gamma)$.

AMS (MOS) subject classifications (1970). Primary 46G10.

Key words and phrases. Weak order unit, band projection, weakly compactly generated Banach space, unconditional constant. 
Note. The hypothesis that $E \in L$ is crucial; e.g., consider the case $E=$ $l_{2}\left(2^{N}\right)$.

3. COROLlaRY. If $E$ is a Banach lattice with a weak order unit, then either $E^{\prime}$ has a weak order unit or $E^{\prime}$ contains a lattice isomorph of a nonseparable $l_{1}(\Gamma)$ space.

4. REMARK. It is possible that $E$ and $E^{\prime}$ both have a weak order unit and $E^{\prime}$ contains a complemented isomorph (but not a lattice isomorph) of $l_{1}\left(2^{N}\right)$. For example, consider $E=\left(\Sigma l_{\infty}(n)\right)_{l_{1}}$. (See [2] for details.)

5. REMARK. If $E$ is a Banach lattice, then condition (i) can be restated in terms of absolute continuity:

(i') For every $\epsilon>0$, every $x \in E_{+}$, and every $a^{\prime} \in \Sigma(A)$, there is a $\delta=$ $\delta\left(\epsilon, x, a^{\prime}\right)>0$ such that $|y| \leqslant x, u^{\prime}(|y|)<\delta$, implies $\left|a^{\prime}(y)\right|<\epsilon$.

6. REMARK. If $E \in L$ and $E$ does not contain a complemented copy of $l_{1}$, then $E^{\prime}$ does not contain a copy of $c_{0}$ (see [6, Corollary 1.2]) and thus (see [7, Theorem 3.7]) each order interval of $E^{\prime}$ is weakly relatively compact. In this case (i) can be restated as follow:

(i") $A$ is contained in a weakly compactly generated (complemented) sublattice having a weak order unit.

As a consequence we retrieve Lemma 1.3 in [6].

Recall that a Banach space $Z$ has local unconditional structure (1.u.st.) if there exists a positive $\lambda>0$ and a directed net $\left\{Z_{a}\right\}_{a}$ of finite dimensional subspaces such that $Z=\bigcup Z_{a}$ and each $Z_{a}$ has a basis whose unconditional constant is $\leqslant \lambda$. The usual Banach spaces all have 1.u.st. We next extend a well-known result due to James for Banach spaces with an unconditional basis.

7. Theorem. For a separable Banach space E having l.u.st., the following assertions are equivalent:

(a) $E^{\prime}$ has the Radon-Nikodym property (i.e., every integral operator from a space $C(S)$ into $E^{\prime}$ is nuclear);

(b) E does not contain an isomorph of $l_{1}$;

(c) $E^{\prime}$ is weakly compactly generated.

Therefore, if $E$ is a separable Banach space with l.u.st., then $E^{\prime}$ is separable if and only if $E$ contains no isomorph of $l_{1}$.

If $Z$ has 1.u.st. and $Y$ is a separable subspace of $Z$, then there is a separable Banach space $X$ with 1.u.st. such that $Y \subset X \subset Z$. (See the proof of Lemmas 3.1 and 3.2 in [4] .) Consequently, the equivalence (a) $\Longleftrightarrow$ (b) holds also in the nonseparable case.

The implications (c) $\Rightarrow(b) \Rightarrow$ (a) are well known. We next prove that (b) $\Rightarrow(c)$. By virtue of Theorem 2.1 and Corollary 2.2 in [1], there exists a separable Banach lattice $L \supset E$ and an isomorphism $\phi$ from $E^{\prime}$ into $L^{\prime}$ such that $i^{\prime} \circ \phi=$ $1_{E^{\prime}}{ }^{\prime}$, where $i: E \rightarrow L$ denotes the canonical embedding. A result due to Pełczyński 
and Hagler (see Studia Math. 46 (1973), 35-42) implies that $E^{\prime}$ does not contain a copy of $l_{1}(\Gamma)$, for $\Gamma$ an uncountable set, so by our Theorem 2 above, $\phi\left(E^{\prime}\right)$ is contained in the band generated by a positive $u^{\prime} \in L^{\prime}$. We conclude by using a technique due to H. P. Lotz. For each $x^{\prime} \in L^{\prime}, x^{\prime} \geqslant 0$, let us denote by $I_{x^{\prime}}$, the ideal generated by $x^{\prime}$ and normed by $\| 1|\cdot|||=\inf \left\{\lambda>0 ; 1 \cdot \mid \leqslant \lambda x^{\prime}\right\}$. Then $I_{x^{\prime}}$, is lattice isometric to a $C(S)$ space. By Corollary 1.2 in [6], $E^{\prime}$ does not contain a copy of $c_{0}$ and thus the composition $j_{x^{\prime}}: I_{x^{\prime}} \rightarrow L^{\prime} \stackrel{i^{\prime}}{\rightarrow} E^{\prime}$ is weakly compact (see [7, Theorem 3.7]). Since $I_{x^{\prime}}$ has the Dunford-Pettis property, $j_{x^{\prime}}$ maps decreasing sequences of positive elements of $I_{x^{\prime}}$ into convergent sequences of elements of $E^{\prime}$. Since $j_{x^{\prime}}$ is $w^{\prime}$-continuous (in fact $j_{x^{\prime}}$ is an adjoint), we obtain that $j_{x^{\prime}}$ is order $\sigma$-continuous for each $x^{\prime} \geqslant 0$. Then $E^{\prime}=$ $\overline{\operatorname{Span}} i^{\prime}\left[0, u^{\prime}\right]$. Q. E. D.

ADDED IN PROOF. We have learned that Corollary 3 above is known (for separable Banach lattices) to H. P. Lotz and to H. Rosenthal.

\section{References}

1. T. Figiel, W. B. Johnson and L. Tzafriri, On Banach lattices and spaces having local unconditional structure with applications to Lorentz function spaces, J. Approximation Theory (to appear).

2. J. Hagler and C. Stegall, Banach spaces whose duals contain complemented subspaces isomorphic to $C[0,1]$, J. Functional Analysis 13 (1973), 233-251. MR 50 \# 2874.

3. R. C. James, $A$ separable somewhat reflexive Banach space with nonseparable dual, Bull. Amer. Math. Soc. 80 (1974), 738-743.

4. J. Lindenstrauss, Extension of compact operators, Mem. Amer. Math. Soc. no. 48, 1964. MR 31 \# 3828.

5. G. Ja. Lozanovskir, Some topological properties of Banach lattices and reflexivity conditions for them, Dokl. Akad. Nauk USSR 183 (1968), 521-523 = Soviet Math. Dokl. 9 (1968), 1415-1418. MR 38 \# 3710.

6. H. P. Rosenthal, On injective Banach spaces and the spaces $L^{\infty}(\mu)$ for finite measures $\mu$, Acta Math. 124 (1970), 205-248. MR 41 \# 2370.

7. On relatively disjoint families of measures, with some applications to Banach space theory, Studia Math. 37 (1970), 13-36. MR 42 \# 5015.

INSTITUTE OF MATHEMATICS, ACADEMIEI 14, BUCHAREST, ROMANIA 